\title{
Mechanical Oscillations of Magnetic Strips under the Influence of External Field
}

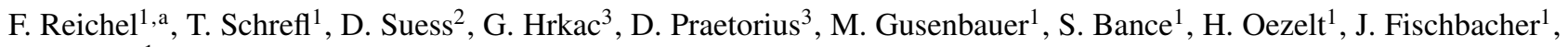 \\ and L. Exl ${ }^{1}$ \\ 1. University of Applied Science, Industrial Simulation, Matthias Corvinus-Strasse 15, 3100 St.Poelten, Austria \\ 2. Vienna University of Technology, Institute of Solid State Physics (Inst. E 138), Wiedner Hauptstrasse 8-10, 1040 Wien, \\ Austria \\ 3. Vienna University of Technology, Institute for Analysis and Scientific Computing (Inst. E 101), Wiedner Hauptstrasse \\ 8-10, 1040 Wien, Austria
}

\begin{abstract}
By application of a magnetic field on an amorphous metallic strip, the orientation of magnetization of Weiss domains can be changed. When the strip changes its length, this effect is called magnetostriction. We simulate this effect using a finite element method. In particular we calculate the change of the mechanical resonance frequency of a magnetic platelet as a function of the applied field. This gives a quantitative model of the influence of the applied magnetic field on the effective Young's Modulus of the material.
\end{abstract}

\section{Introduction}

In-plane Weiss domains occur in soft ferromagnetic ribbons as a consequence of the minimization of the Gibbs free energy. The formation of magnetic domains reduce the magneto-static energy at the expense of the wall energy. In magnetic platelets exceeding the single domain limit magnetic domains are always present. These domains can be reoriented by applying an external magnetic field. Owing to the magnetoelastic coupling energy a change in magnetization changes the length of the ribbon. Conversely, a change of length of the ribbon changes the orientation of the magnetization in the magnetic domains. Mechanical vibrations lead to a periodic change of the magnetization of the ribbon. The oscillation frequency shifts with an applied magnetic DC field. We compute this frequency shift for the second harmonic of the longitudinal oscillation.

The magnetostrictive effect is especially effective if the magnetic field is applied perpendicular to the easy axis as shown in Fig.1.

\section{Equations}

In an one-dimensional model the oscillations of a magnetic strip are given by the following equation

$$
\rho \frac{\partial^{2} u}{\partial t^{2}}=\frac{\partial \sigma}{\partial x}
$$

Here $u$ is the displacement, $\sigma$ is the stress, $\rho$ is the density of the material. $t$ and $x$ are the time and space coordinates. The stress is given by Hook's law as:

$$
\sigma=E \epsilon^{e}
$$

\footnotetext{
a e-mail: franz.reichel@fhstp.ac.at
}

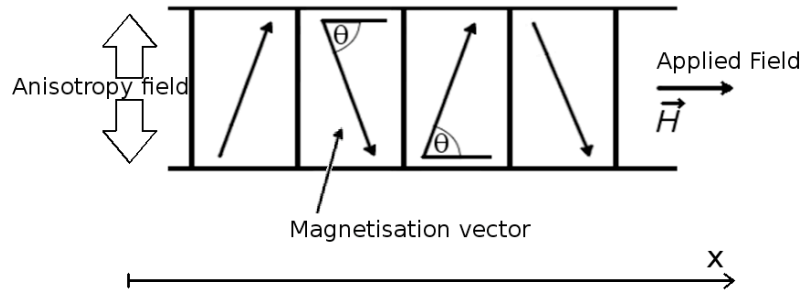

Fig. 1. Orientation of magnetization of Weiss domains on a metallic strip. The magnetization angle $\phi$ is defined as the angle between local magnetization vector $J$ and the magnetization field $H$. A transverse induced anisotropy creates the perpendicular domain pattern.

where is $E$ is the Young's Modulus and $\epsilon^{e}$ is the elastic contribution of the strain, which causes a change in stress. Similar to the thermal strain the magnetic strain, $\epsilon^{m}$ does not change the stress. The total strain is the sum of the magnetic and elastic contribution.

$$
\epsilon=\epsilon^{m}+\epsilon^{e}
$$

According to [3] in an isotropic material the magnetic strain is:

$$
\epsilon^{m}=\frac{3 \lambda_{s}}{2}\left(\cos ^{2} \theta-\frac{1}{3}\right)
$$

Here $\theta$ is the angle of the magnetization with respect to the $x$ axis (long axis of the magnetic strip). $\lambda_{s}$ is the magnetostriction constant.

The total strain $\epsilon$ is given as the partial derivative of the displacement $u$ with respect to $x$

This is an Open Access article distributed under the terms of the Creative Commons Attribution License 2.0, which permits unrestricted use, distribution, and reproduction in any medium, provided the original work is properly cited. 


$$
\epsilon=\frac{\partial u}{\partial x}
$$

Using the above equations (3)-(5) we can rewrite Hook's law as:

$$
\sigma=E\left(\epsilon-\epsilon^{m}\right)=E\left(\frac{\partial u}{\partial x}-\frac{3 \lambda_{s}}{2}\left(\cos ^{2} \theta-\frac{1}{3}\right)\right)
$$

Replacing $\sigma$ in equation (1) with (6) gives

$$
\rho \frac{\partial^{2} u}{\partial t^{2}}=\frac{\partial}{\partial x} E\left(\frac{\partial u}{\partial x}-\frac{3 \lambda_{s}}{2}\left(\cos ^{2} \theta-\frac{1}{3}\right)\right)
$$

This is the one-dimensional wave equation for the longitudinal mechanical oscillation of a magnetostrictive strip.

The right-hand side of equation (7) contains the magnetomechanical coupling that links the magnetization angle $\theta$ with the mechanical displacement. For the simulations it is convenient to non-dimensionalize the variables in time and space.

We define

$$
x^{\prime}=\frac{x}{L}
$$

where $L$ is the length of the ribbon and

$$
t^{\prime}=\sqrt{\frac{E}{\rho}} * \frac{1}{L} t
$$

For this transformation we assume that the Young's Modulus is uniform within the sample. Then the equation of motion reads:

$$
\frac{\partial^{2} u}{\partial t^{\prime}}=\frac{\partial^{2} u}{\partial x^{\prime 2}}-\frac{\partial}{\partial x^{\prime}}\left(\frac{3 \lambda_{s} L}{2}\left(\cos ^{2} \theta-\frac{1}{3}\right)\right)
$$

This coupling is nonlinear as the magnetization angle depends on the external field $H$ and the local stress $\sigma(x)$ via the following set of equations:

$$
\begin{gathered}
\cos \theta=\frac{H}{H_{A \sigma}} \\
H_{A \sigma}=\frac{2 K_{u}-3 \lambda_{s} \sigma}{J_{s}}
\end{gathered}
$$

$H_{A \sigma}$ is the local anisotropy field which is composed from the magnetocrystalline anisotropy energy and the magnetoelastic coupling energy. Equations (11) and (12) follow from the minimization of the magnetic energy:

$$
E(\theta)=\left(K_{u}-\frac{3}{2} \lambda \sigma\right) \cos ^{2} \theta-H J_{s} \cos \theta
$$

Here the first term is the net anisotropy energy which is composed of the magnetocrystalline anisotropy energy with the magnetocrystalline anisotropy constant $K_{u}$ with the magnetoelastic coupling energy due to a longitudinal stress $\sigma$.

The second term is the Zeeman energy of the magnetization in the external field. Here we assume that the domain structure is fixed and the application of a field just changes the orientation of the magnetization in the domains (see Fig.1 and ref [3]).

For the numerical treatment of the problem we apply the method of lines: We apply the Galerkin method, in order to discretize equation (7) in space. The shape and basis functions are piecewise linear polynomials. This results in $N$ second order differential equations in time, where $N$ is the number of nodes in the finite element mesh.

$$
M_{i j} \frac{\partial^{2} u_{i j}}{\partial t^{\prime 2}}+A_{i j} u_{j}=f_{i}(\cos \theta), \quad i=1, \ldots, N
$$

$M_{i j}$ is the finite element Mass Matrix [4], $A_{i j}$ is the finite element Stiffness matrix [4] and the load vector $f_{i}$ is given as:

$$
f_{i}=\int \frac{\partial N_{i}}{\partial x} \frac{3 \lambda_{s}}{2}\left(\cos ^{2} \theta-\frac{1}{3}\right) d x
$$

where $N_{i}$ is the finite element basis function of node $i$.

We transform these equations into corresponding set of $2 \mathrm{~N}$ ordinary differential equations. We solve the system of equations with the Adams Bashforth method as provided by the software library "SUNDIALS" [5].

$$
\begin{gathered}
\frac{\partial u_{i}}{\partial u}=v_{i}, \quad i=1, \ldots, N \\
M_{i j} \frac{\partial v_{j}}{\partial t^{2}}+A_{i j} u_{j}=f_{i}(\cos \theta), \quad i=1, \ldots, N
\end{gathered}
$$

The right-hand side of equation (17) contains the magnetization angle $\theta$ which depends on the applied field and stress $\sigma$. For solving the set of ordinary differential equations with sundials [5] we have to provide the right-hand side of equation (15). For evaluating $f_{i}$ we apply a fixed point iteration to calculate $\cos \theta$.

Table 1. Fixed point scheme for computing $\cos \theta$

$\begin{aligned} \text { for }(n & =0,1,2, \ldots) \\ & \text { compute }\left(\frac{\partial u}{\partial x}\right)_{n} \\ & \text { compute } \sigma_{n} \text { from equation (6) } \\ & \text { compute } \cos \theta_{n} \text { from equations (11) and (12) }\end{aligned}$

\section{Results}

In the simulations we compute $u=u(x, t)$ by integrating equation (7) numerically, whereby we augmented the right-hand side with a damping term $-\lambda d u / d x$. From the analysis of $u_{i}=u\left(x_{i}, t\right)$ we can compute the frequency of the oscillations, here $i$ is the node index of the finite element mesh.

As the initial state of the simulation we use the second harmonic of a longitudinal standing wave. The initial displacement $u(x, t=0)$ is shown in Fig. 2. Starting from this initial condition we integrate the equation of motion in time. Figure (3) shows the displacement, $u_{i}$, of selected nodes of the finite element mesh. 


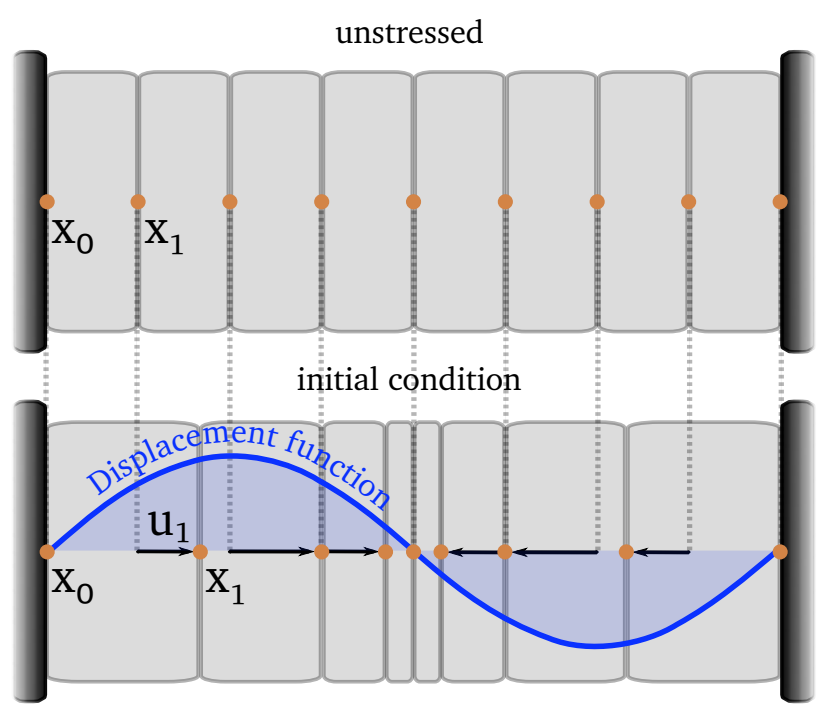

Fig. 2. The upper part of the picture shows an unstressed metallic strip with equidistant measuring points $x_{i}$. The lower part shows this metallic strip with the displacement function superimposed for the initial conditions of the simulations $(t=0)$.

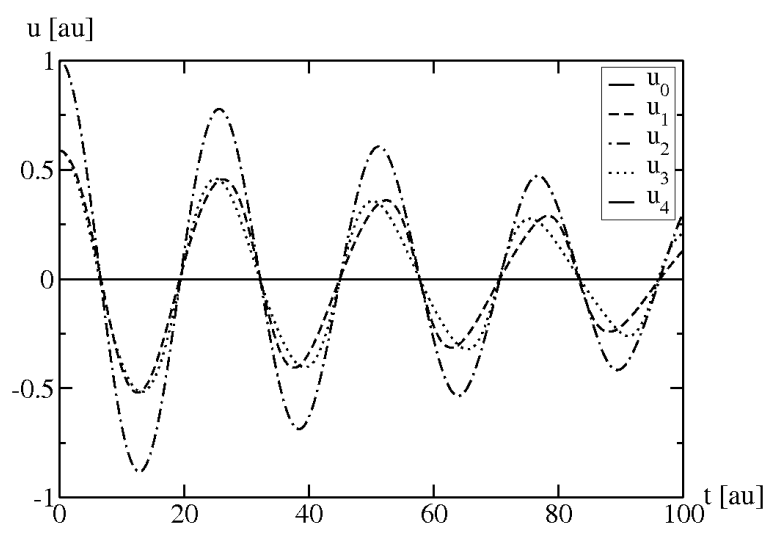

Fig. 3. Displacement $u$ of selected nodes as function of time $t$. The first point $u_{0}$ and the last point $u_{n}$ are fixed and therefore zero at every timestep. Same for $u_{4}$ because it is the root of the displacement function.

From the Fourier transform of $u_{i}(t)$ we compute the oscillation frequency (see Fig. 4.a). This procedure was applied for different external fields. Fig. 4.b shows the change in oscillation frequency with the applied field. The frequency decreases with increasing external field. This effect is known as the " $\Delta E$ effect" [3]. This effect is used in magnetic tapes for article surveillance [6].

\section{Conclusion}

A finite element method for the simulation of the mechanical vibrations of magnetostrictive platelets was developed. With this method we computed the $\Delta E$ effect for simple transverse domain structures. This is the first step for a dynamic elastic-micromagnetic solver that will compute the mechanical response of magnetostrictive samples by application of an excitation field. In the future we will replace

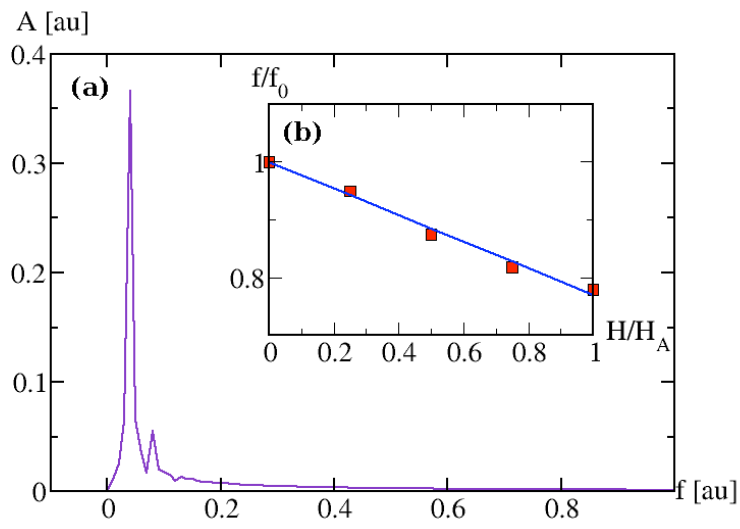

Fig. 4. a) Fourier transform $u_{i}(t)$ for $i=7$ of the displacement of a specific node.

b) Change of oscillation frequency with respect to the external field. $f_{0}$ is the frequency with zero external field. $H_{A}$ is the anisotropy field. The axes are normalized by $f_{0}$ and $H_{A}$.

equation (11) with a micromagnetic solver for the computation of the magnetization distribution.

\section{References}

1. Lúbomír Ban̆as, On dynamical Micromagnetism with Magnetostriction (University of Gent, PhD Thesis June 2005) 142

2. Craig A. Grimes, Sensors and Actuators 84, (2000) 205-212

3. J.D.Livingston, Phys. Stat. Sol. (a) 70, (1982) 591

4. Bernhard Bergmair, Applied Physics Letters 101, 042412 (2012)

5. Thomas Huber, Applied Physics Letters 101, 042402 (2012)

6. Giselher Herzer, Journal of Magnetism and Magnetic Materials 254-255, (2003) 598-602

7. Gennadiy Nikishkov, Programming Finite Elements in Java (Springer Verlag, 2010) 402

8. A.C. Hindmarsh, P.N. Brown, K.E. Grant, S.L. Lee, R. Serban, D.E. Shumaker, C.S. Woodward, Sundials: Suite of nonlinear and differential/algebraic equation solvers (ACM Transactions on mathematical software, 31 (3), 2005) 363-396 